\title{
ARTHROSCOPIC SURGERY OF THE SHOULDER
}

\author{
A GENERAL APPRAISAL
}

D. J. OGILVIE-HARRIS. A. M. WILEY

From Toronto Western Hospital and the University of Toronto

\begin{abstract}
Arthroscopic surgery of the shoulder was performed on 439 patients over a 10-year period: these patients are reviewed after a minimum follow-up of one year. Diagnostic arthroscopy is known to be valuable and we have found that arthroscopic surgery also is safe and effective. It was useful in treating frozen shoulder, early osteoarthritis, isolated tears of the glenoid labrum and lesions of the biceps tendon. It was less useful in treating partial tears of the rotator cuff, tendonitis and severe osteoarthritis, and of little value in treating complete tears of the rotator cuff or in treating patients in whom previous operations on the rotator cuff had failed. It may prove to be a useful method of performing synovectomy in rheumatoid arthritis and of treating instability.
\end{abstract}

Over the past 10 years arthroscopy of the shoulder has become a standard diagnostic procedure in our unit. During this time over 800 arthroscopies have been performed and 439 of these patients had arthroscopic surgical procedures. These have been reviewed in order to assess the results and to determine which conditions might benefit from arthroscopic surgery.

\section{MATERIALS AND METHODS}

The arthroscopies were carried out under general anaesthesia usually as an outpatient: only if there were anaesthetic or medical indications were patients admitted.

The arthroscopy was performed with the patient lying on the unaffected side and the trunk elevated approximately 30 from the horizontal. No mechanical arm holder was used, but traction was applied to the affected arm by an assistant. Before inserting the arthroscope the shoulder was distended with fluid and its capacity noted. The arthroscope, usually a $4 \mathrm{~mm}$ model, was introduced posteriorly, approximately $2.5 \mathrm{~cm}$ below the spine of the scapula and $5 \mathrm{~cm}$ medial to the tip of the acromion; it entered the joint through the posterior capsule. Figure 1 shows the structures routinely visualised.

The surgical procedures were performed through accessory posterior or anterior portals. The anterior portal was usually selected under direct vision across the joint so that the instrument could be introduced just below the intra-articular portion of the biceps tendon. A

D. J. Ogilvie-Harris, FRCS(C). Orthopaedic Surgeon

A. M. Wiley. FRCS(C). Orthopaedic Surgeon

Division of Orthopaedic Surgery. University of Toronto and Toronto Western Hospital, 399 Bathurst Street. Toronto, Canada M5T 2S8.

Requests for reprints should be sent to Dr D. J. Ogilvie-Harris. Suite 311. 25 Leonard Avenue. Toronto. Canada M9A 2R2

( 1986 British Editorial Society of Bone and Joint Surgery $0301620 \times 862133 \$ 2.00$ superior portal in the interval between the acromion and clavicle was often used for drainage.

A standard probe was used to feel the structures of the shoulder. Other instruments included straight and curved varieties of basket forceps, pituitary forceps and small scissors. Powered instruments included burrs and shavers; specially designed screws were used for instability.

Detailed notes had been kept and if the patient had been seen within the last six months this information was considered acceptable. Other patients were followed up by phone, letter, or by reviewing the charts. All patients who had had arthroscopic surgery in the 10 years between 1973 and 1983 were followed up. so that the minimum follow-up was one year. Fifty-two patients had

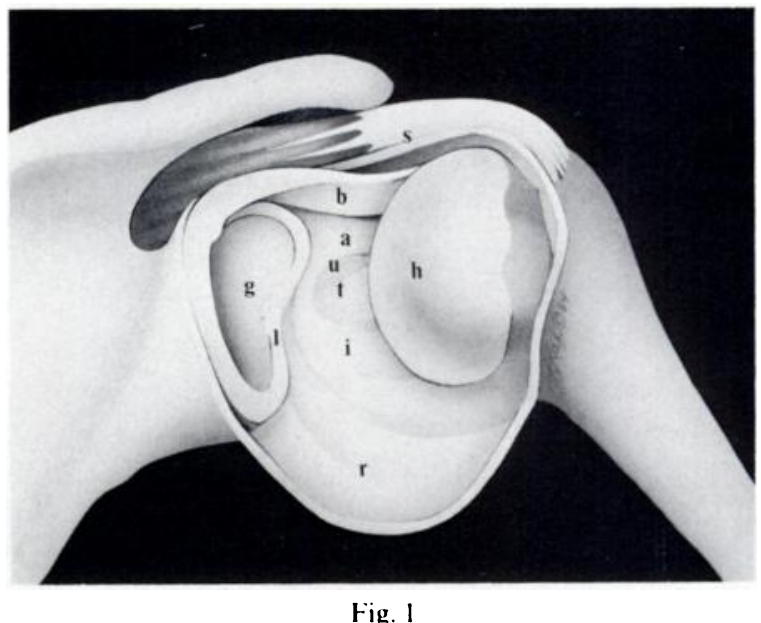

Typical structures that may be visualised routinely at arthroscopy: $g$. glenoid: $l$. glenoid labrum: $h$. biceps tendon: $h$. humeral head: $s$, undersurface of supraspinatus: $t$, subscapularis tendon: $u$. subscapularis bursa: $r$, infraglenoid recess; $a$. superior glenohumeral ligament: $i$, inferior glenohumeral ligament. 
inadequate data or could not be contacted, leaving a total of 439 patients in which there was adequate information for review.

\section{Assessment of results}

In view of the lack of standard methods of reporting shoulder assessment we decided to use a relatively simple system. Our criteria for success were that the symptoms had improved significantly; the patients had little or no pain, were able to perform most of the activities they wished (though not necessarily all activities) and had a functional range of movement such that the hand on the affected side could be placed behind the neck, behind the back and above the head. Patients not meeting all these criteria were considered failures. It should be appreciated therefore that a successful result does not necessarily mean that the patient has been restored to normal nor does a failure mean that there has been no improvement. We felt. however, that a more detailed assessment would be inaccurate and might be misleading; it may, however, be possible and worth while in a prospective study.

\section{RESULTS}

There were three major problems in presenting the results; first, not all patients were reviewed in person; secondly, the study was retrospective; and thirdly, some of the conditions described do not lend themselves to precise definition. Consequently, it was felt inappropriate to express our results in precise numerical terms. The series, however, seemed large enough for our impressions to be considered useful and these are discussed according to the pathology found at arthroscopy. Frozen shoulder. This was defined as a stiff painful shoulder which had developed spontaneously with no recognisable pathological cause. The patients had not improved despite physiotherapy for six months. They had limited abduction and rotation, and movement could not be improved by injecting local anaesthetic into or around the shoulder joint. The joint capacity was found at arthroscopy to be significantly decreased, often being $20 \mathrm{ml}$ or less.

In most such joints it was possible to see mild synovitis. The infraglenoid recess was visible and was not obliterated by adhesions. However, there appeared to be extrasynovial contracture of the soft tissue; in most cases it was the anterior structures which were particularly tight. On laterally rotating the shoulder one could see the anterior capsular structures pulled tightly across the front of the humeral head and this was the major cause of the limitation of movement.

In the early part of our series we distended the shoulder joint fully and this produced a capsular rupture usually in the anterior and inferior portion of the capsule. The shoulder was then manipulated through its full range of movement. After manipulation it was apparent that the anterior structures had been torn and it was possible with the arthroscope to look through the synovium and capsule and into the subscapularis tendon and muscle; in addition, there was often a tear of the infraglenoid recess. It is apparent, therefore, that the effect of the manipulation and distension in these patients was to break down the extrasynovial soft-tissue contracture. In the later portion of the series we performed this freeingup procedure with a blunt instrument or, in some cases, by cutting the anterior capsule progressively and improving the movement in a controlled manner. Postoperatively, the patients started exercises immediately, either on their own or with the help of a physiotherapist.

Eighty-one patients with frozen shoulder were treated and the average follow-up was just under four years. Approximately half the cases were idiopathic and uncomplicated but in 14 there was also mild osteoarthritis and in 17 a partial tear of the rotator cuff. In general successful results were achieved. It is of course acknowledged that most patients with uncomplicated frozen shoulder recover even without treatment, but after our procedures they recovered more rapidly than we would have expected without intervention. Eleven of our 81 patients were diabetic and in them the results were less satisfactory.

Rotator cuff lesions (Figs 2 and 3). Patients who came to arthroscopic surgery after rotator cuff tears or tendonitis had first had at least six months of conservative management to which they had failed to respond. The arthroscopic procedure consisted simply of debridement of the rotator cuff, using powered instruments or hand tools. Often there was a significant amount of debris; it had broken off from the cuff and was evacuated from the joint by irrigation, often using suction. Postoperatively the patients were mobilised rapidly, either by exercising themselves or with the help of physiotherapy.

There were 174 patients with lesions of the rotator cuff and their average follow-up was three years. Of these, 17 also had a frozen shoulder, seven had osteoarthritis and nine had lesions of the biceps tendon; these associated conditions are dealt with under their appropriate sections. Fifty-seven patients had partial tears and 36 had complete tears. Twenty patients had tendonitis of the rotator cuff. Eight had had repairs of a torn rotator cuff which had subsequently re-ruptured and 20 patients had had previous unsuccessful open operations leaving them with continuing pain and restricted movement.

The best results were achieved with rotator cuff tendonitis, approximately two out of three being successful. With partial tears and re-tears approximately half the patients had successful results. The results were poor in patients who had had a complete tear and in those who had had previous operations on the rotator cuff.

Osteoarthritis (Figs 4 and 5). Patients with osteoarthritis of the shoulder were divided into those in whom it was mild, with damage only to the superficial areas of articular cartilage, and those in whom it was severe, with exposure of the subchondral bone. At arthroscopy debris and 


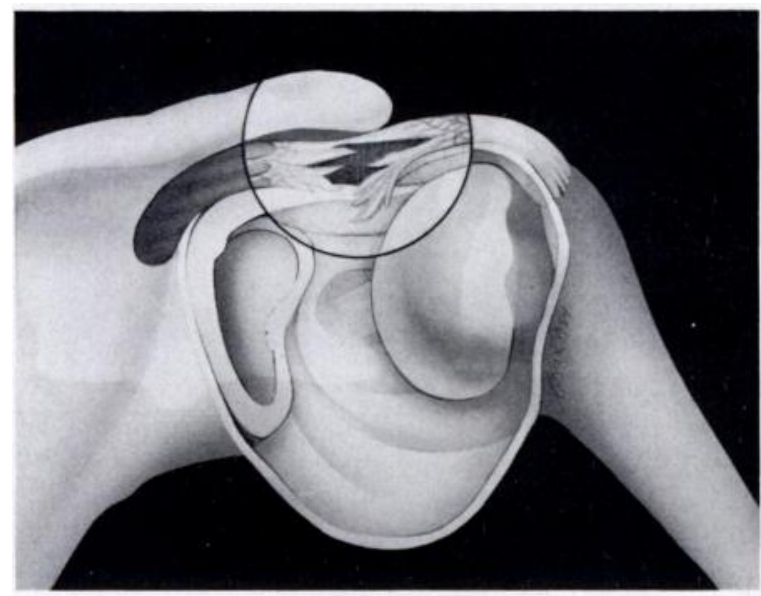

Fig. 2

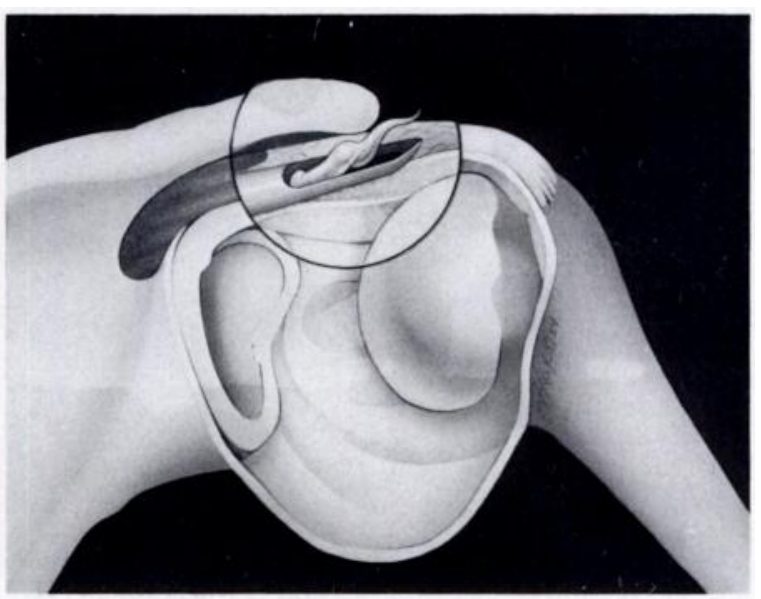

Fig. 3

Figure 2-There is a partial tear of the supraspinatus tendon with fronds dangling down. Figure 3 Debridement of the rotator cuff with a power shaver.

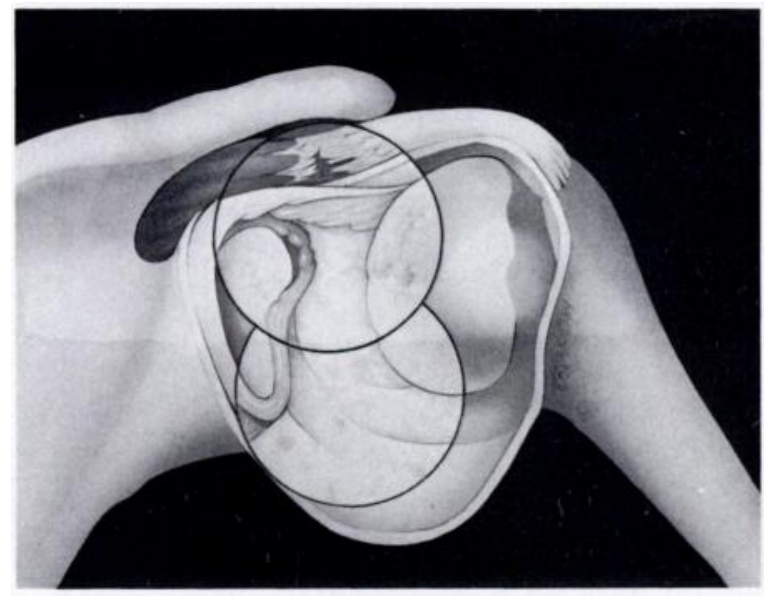

Fig. 4

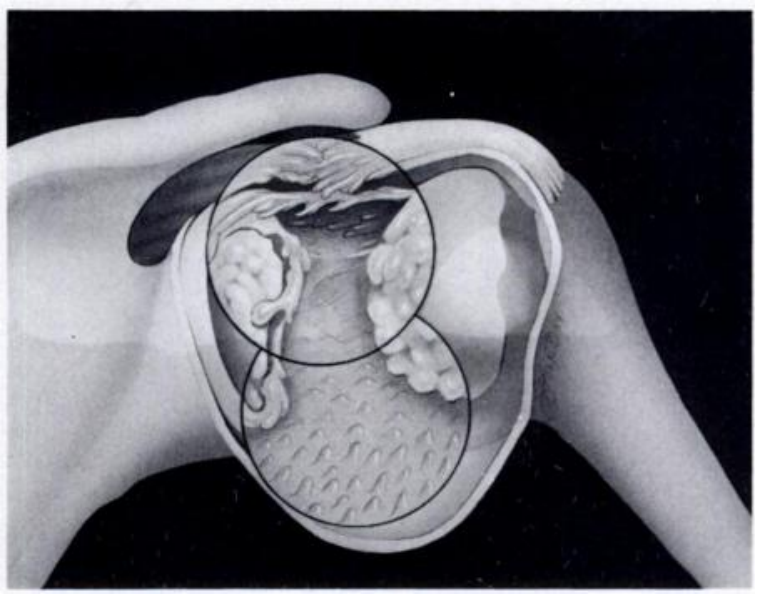

Fig. 5

Figure 4-Osteoarthritis of the shoulder, mild. There are degenerative chondral changes of the glenoid and humeral head. Mild changes are seen in the biceps tendon and a small tear in the rotator cuff. Figure 5 - Osteoarthritis of the shoulder. severe. There is a diffuse synovitis. Marked degenerative changes are seen in the glenoid, glenoid labrum, humeral head, biceps and rotator cuff. 


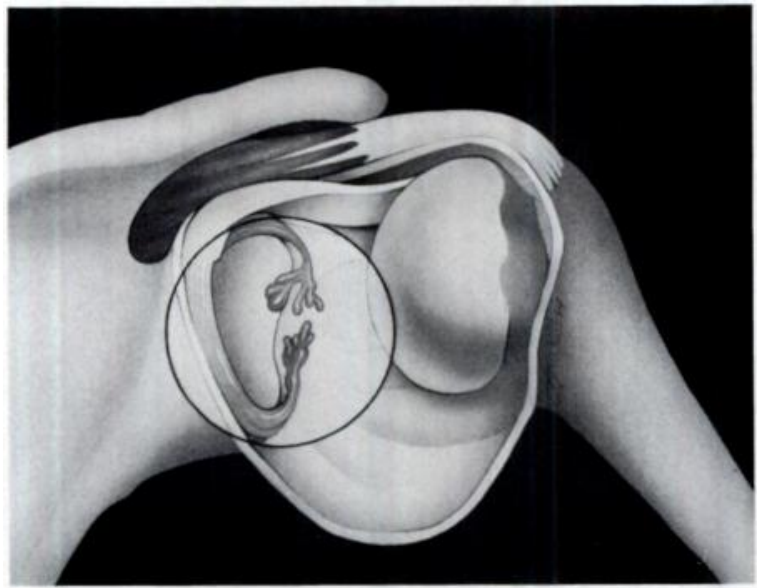

Fig. 6

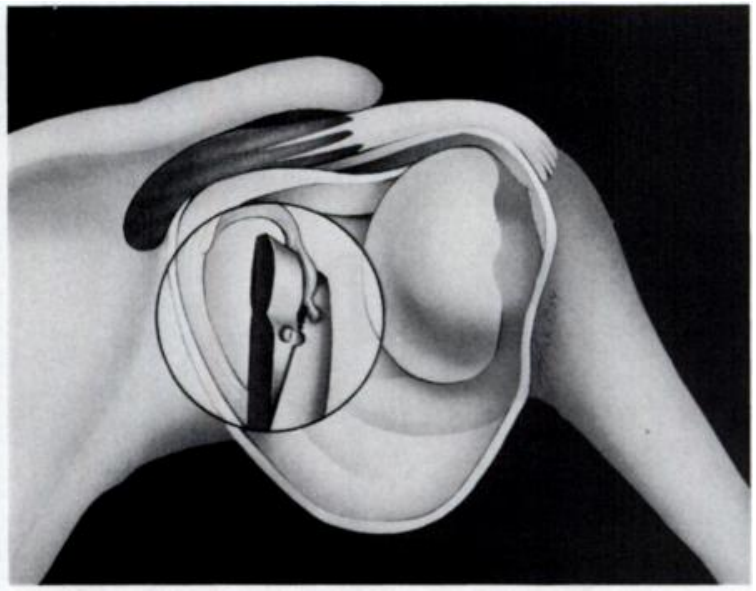

Fig. 7

Figure 6-Isolated tear of the glenoid labrum: unstable. Figure 7 -Excising torn portion of glenoid labrum.

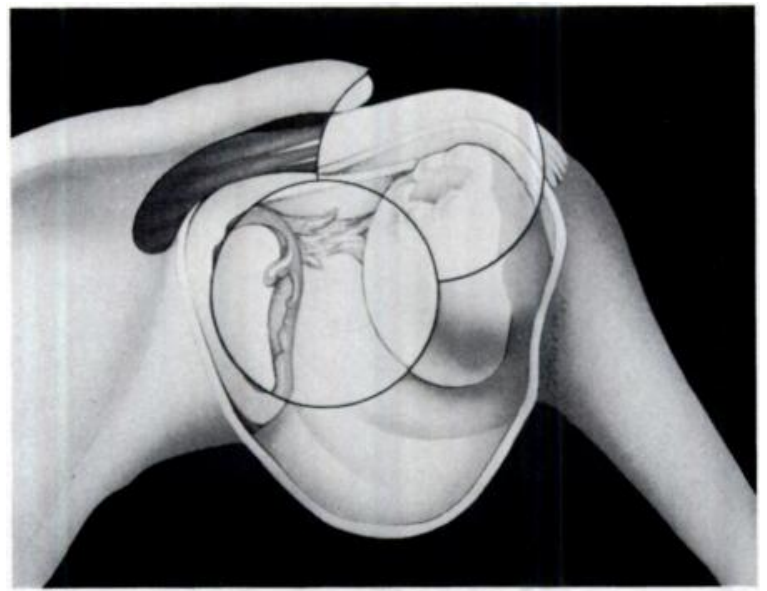

Fig. 8

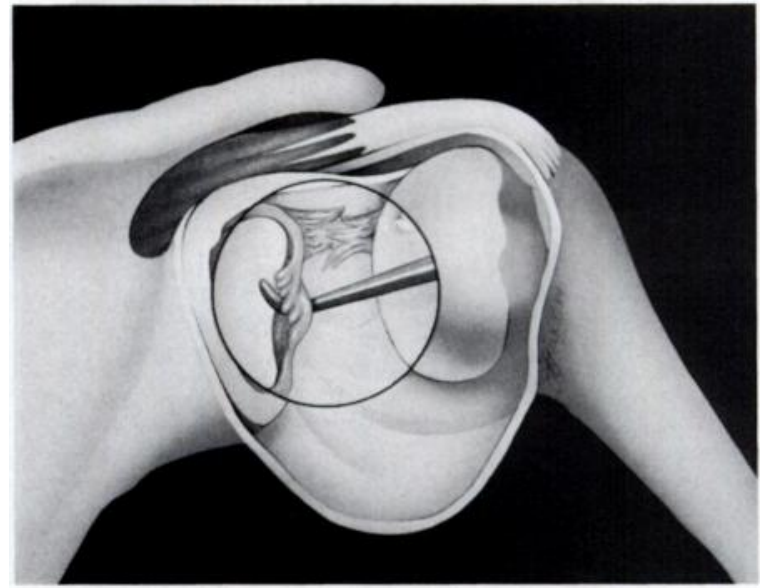

Fig. 9

Figure 8 Typical findings in the unstable shoulder: torn. detached glenoid labrum with Bankart lesion: cartilaginous Hill Sachs lesion: and torn glenohumeral ligament. Figure 9. Probing the torn glenoid labrum: this demonstrated the anterior capsular detachment representing the Bankart lesion.

chondral fragments were removed from the joint and any abnormal masses of synovium were resected. If movement was significantly restricted it was restored either by cutting and removing adhesions, or by manipulation. Major co-existing pathology was found in many shoulders: 14 patients had an associated frozen shoulder, three had loose bodies, three had instability of the shoulder in an anterior direction, five had major pathology in the biceps tendon and seven had tears of the glenoid labrum. Each of these conditions was dealt with in addition to the debridement.

There were 54 patients with osteoarthritis of the shoulder, and the follow-up was approximately three years. When the degeneration was mild, successful results were achieved in about two-thirds of cases; in severe cases only one-third had good results. The patients who also had a frozen shoulder seemed to bene- fit particularly and this is probably because, not only was the osteoarthritic debris removed, but also the range of movement was restored. Patients with a glenoid labrum tear also seemed to do well; this lesion may well be analogous to a degenerative tear of the meniscus in an osteoarthritic knee.

Glenoid labrum tears and instability (Figs 6. 7.8 and 9). We used the term "instability" of the shoulder to include anterior dislocation, anterior subluxation and multidirectional instability; this seemed to us more useful than referring to each condition separately. Instability was determined by examination under general anaesthesia.

At arthroscopy tears of the glenoid labrum were easily visualised and probed. Tears of the anterosuperior one-third of the labrum seemed to be associated with a clicking noise in the shoulder on physical examination and often with adhesions and evidence of inflammation 
around the attachment of the biceps tendon to the glenoid tubercle. Sometimes the tear was in the main body of the labrum directly anterior to the glenoid; here it was often associated with stripping of the capsule from the anterior surface of the neck of the glenoid. or with a Bankart lesion. With either of these lesions we resected the torn portion of the labrum.

In the presence of instability of the shoulder there were classic arthroscopic findings. The Bankart lesion could often be found as mentioned earlier. There was usually a defect in the posterosuperior aspect of the humeral head. the Hill Sachs lesion: this defect was often cartilaginous rather than bony and sometimes there was simply a chondral ulcer in the area where the bony defect is usually seen on the radiograph. In three cases loose bodies were found in the joint.

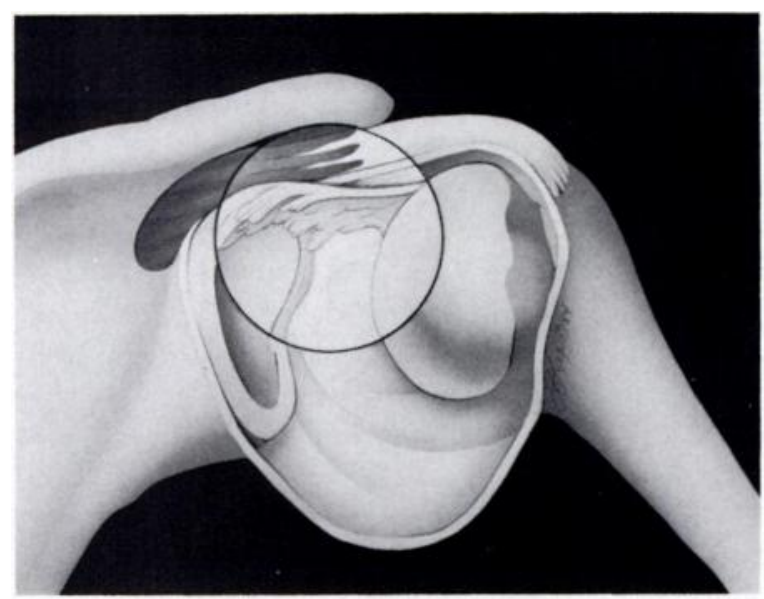

Fig. 10

Bicipital tendon lesions. Frond formation along the tendon is seen. The glenoid tubercle is elevated and surrounded by adhesions.

Twenty-three patients had stable shoulders with isolated tears of the glenoid labrum. almost always in the upper third: 32 had tears which were associated with instability of the shoulder. Forty-five patients had instability of the shoulder without a tear in the labrum. Seven patients had osteoarthritis in association with a tear.

In stable shoulders, tears of the glenoid labrum were resected and this was successful in relieving the symptoms. In the presence of instability, however, resection of a labral tear produced a poor result; most patients continued to have symptoms or became worse and needed operative stabilisation. We therefore consider that, in the presence of instability, resection of the glenoid labrum is contra-indicated.

Of the patients with osteoarthritis of the shoulder and a glenoid labrum tear. approximately two-thirds had relief of their symptoms.

Biceps tendon lesions (Fig. 10). The usual lesion consisted of fraying of the tendon with loose fronds hanging down into the joint. The fraying appeared to be greatest at the point where the biceps tendon entered the bicipital groove. The arthroscopic procedure consisted of debridement of the tendon by removing all the loose fronds. In some cases we attempted to dilate the orifice through which the biceps tendon passed.

There were 46 patients with lesions of the biceps tendon: nine of these had had previous rotator cuff repairs and in them the biceps tendon appeared to be adherent to the undersurface of the cuff tear. We freed the adhesions arthroscopically using small scissors and tried to strip any adhesions off the biceps tendon with pituitary forceps.

In five of the patients there was an associated osteoarthritis: in them we performed a debridement of the biceps tendon as well as a generalised debridement for the osteoarthritis.

Of the patients with isolated lesions of the biceps tendon. about three-quarters did well with simple debridement. However, our follow-up was relatively short, being only 24 months. Of particular interest were the nine patients in whom the biceps tendon was stuck to the undersurface of the rotator cuff after a previous repair: four of these patients had relief of their symptoms as a result of freeing the tendon. Considering the generally poor results of treating failures after rotator cuff operations. we feel that this lesion is particularly worth noting and attempting to treat.

In patients with osteoarthritis and a biceps tendon lesion the results were much the same as those after a generalised debridement for osteoarthritis alone: the biceps tendon lesion. therefore. was probably merely part of the overall pathology of the osteoarthritis.

Loose bodies (Fig. 11). In 11 patients loose bodies were found and were removed using grasping forceps; three had osteoarthritis and three had instability of the shoulder. In 10 of the 11 cases we were able to remove the loose bodies successfully: however. in one case with an unstable shoulder a fairly large loose body could not be

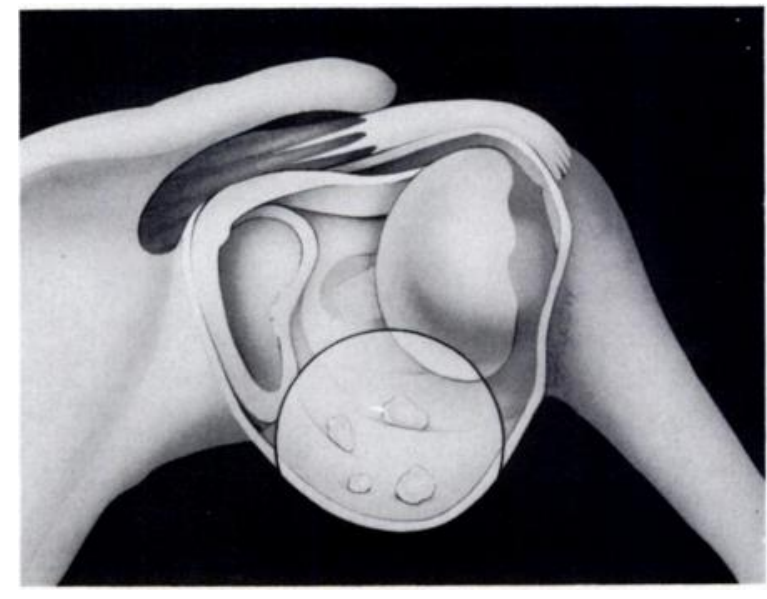

Fig. 11

Loose bodies in the infraglenoid recess. 
extracted arthroscopically and subsequently had to be removed at open operation.

Synovectomy. We had 11 patients with rheumatoid arthritis of the shoulder. These patients did not have radiographic evidence of gross degenerative changes; they did, however, have persistent weakness and swelling of the shoulder that had not responded to conservative management. In these patients an arthroscopic synovectomy was performed using powered instruments. This was often technically difficult because of bleeding and a double irrigation system had to be used. Postoperatively the patients were mobilised and active physiotherapy was instituted.

Of our 11 patients nine had significant improvement, with less pain and increased movement. However, the follow-up was short, averaging only 14 months.

Sepsis. In five patients septic arthritis was found at arthroscopy. The arthroscopic procedure consisted of irrigating the joint thoroughly and we were able to remove a large amount of debris; we did not perform a synovectomy. Suction-irrigation tubes were left in the shoulder and continuous lavage was instituted. The shoulder was mobilised as the patient's pain permitted and appropriate antibiotics were given systemically.

The follow-up in these patients was 32 months. Three of the five patients had restoration of movement and relief of pain. The other two continued to have significant restriction of movement and pain; in both there had been considerable delay in referral which may explain the poor results.

Percutaneous fixation for instability. In eight patients we have performed arthroscopic procedures for anterior instability. First the anterior glenoid margin is roughened, then a screw is introduced percutaneously through the anterior capsule into the anterior aspect of the neck of the glenoid. The patient then wears a sling for six weeks, after which the screw is removed (leaving it in place seems risky).

Our follow-up was approximately 12 months. Although all eight cases have so far been successful, we still have reservations as to the use of this technique.

Complications. Of the 439 patients who had arthroscopic surgery, $3 \%$ had a complication. In eight cases there was massive leakage of fluid from the shoulder joint; it tended to track along the posterior and anterior chest wall and down the arm but. apart from the nuisance value, it presented no problems and subsided within a week. In five patients the articular cartilage on the humeral head or the glenoid cavity was damaged during the arthroscopic procedure; four of these five were patients with frozen shoulders, in which introduction of the arthroscope is difficult. There was one case of postarthroscopic sepsis; the shoulder was drained, suctionirrigation tubes were placed into the joint. antibiotics were given, and recovery was good. There was one case of musculocutaneous nerve palsy after arthroscopy; this resolved in about six weeks leaving no long-term effects.

\section{DISCUSSION}

Arthroscopy of the shoulder as an investigative technique in the management of shoulder conditions has been established as a safe and reasonable procedure (Wiley and Older 1980; Cofield 1983). However, it is only in recent years that arthroscopic surgical procedures have been attempted or carried out with any frequency (Andrews and Carson 1983; Matthews, Vetter and Helfet 1984). We therefore felt it would be of interest to assess the results of our series.

The arthroscopic surgery itself proved to be safe and the incidence of complications was low. Scoring of the articular cartilage, although it occurred in five cases, was minor and probably will not have any ill effects. The incidence of nerve lesions (one case of musculocutaneous nerve palsy) seems to be lower than in other reported series (Andrews and Carson 1983). This may be perhaps because we do not use an arm holder but prefer manual traction: pulling the arm in a longitudinal rather than a vertical direction may decrease the chance of a nerve palsy.

The results in the frozen shoulder series suggest that recovery can be hastened by arthroscopic distension combined with breaking down the contracted tissues (Neviaser 1949; Simmonds 1949: Lundberg 1969; Conti 1979: Ha'eri and Maitland 1981). Fourteen patients had associated mild osteoarthritis and 17 had partial tears of the rotator cuff, but these may well have been incidental since these patients were in the age group where such conditions are not uncommon (Petersson 1983). However, it may be that this type of minor intra-articular pathology could act as a stimulus to the development of extra-articular soft-tissue inflammation and subsequent contracture (Loyd and Loyd 1983). It is interesting to note that those patients with associated intra-articular pathology had a considerably poorer result than those who did not.

With tears of the rotator cuff, in general the results were not good, and with complete tears they were definitely poor; only patients with rotator cuff tendonitis rather than an actual tear did well. We felt that the main reason why tears did badly was that intra-articular debridement does not relieve the extra-articular impingement of the rotator cuff with which they are associated (Ha'eri and Wiley 1981; Earnshaw et al. 1982; Packer et al. 1983). At present we are trying arthroscopic resection of the coraco-acromial ligament but it is too early to assess the results. In patients who have had previous operations on the rotator cuff it is worth looking to see if the biceps tendon is stuck to the undersurface of the cuff, since freeing the tendon seems to relieve the symptoms in about half the patients.

For the unstable shoulder arthroscopy is a very useful diagnostic procedure (Moseley and Övergaard 1962; Pappas, Goss and Kleinman 1983; Matthews et al. 1984). Where there is an associated tear of the glenoid labrum the shoulder needs to be stabilised; at present this 
requires an open operation, but percutaneous screw fixation under arthroscopic control may offer an alternative in the future. A torn glenoid labrum without instability of the shoulder can be treated by resection of the torn portion; this relieves the symptoms and substantially improves function. In an osteoarthritic shoulder with a torn glenoid labrum, again resection of the torn portion seems to improve the condition.

With osteoarthritis per se, general debridement of the shoulder is beneficial providing the osteoarthritis is relatively mild. When there is an associated frozen shoulder, restoration of movement is extremely helpful. Removal of loose bodies, of a torn glenoid labrum and debridement of the biceps tendon also seem to improve substantially the function of a mildly osteoarthritic shoulder.

We were surprised at the degree of success that was achieved following debridement of the biceps tendon. It has often been said that lesions of the biceps tendon are associated with the impingement syndrome (DePalma 1983), so that one would not expect simple debridement of the tendon to relieve symptoms. However, our followup in these cases was only about 24 months and as nothing was done to treat the impingement itself the symptoms may recur. It is, however, possible that in the successful cases the lesion of the biceps tendon was an isolated one (Hawkins and Kennedy 1980).

Conclusions. Arthroscopic surgery seems to be a safe and effective way of treating some conditions of the shoulder; manual traction is recommended in preference to an arm holder.

Successful results can be achieved in the majority of patients with frozen shoulder, rotator cuff tendonitis, tears of the glenoid labrum without instability, lesions of the biceps tendon and mild osteoarthritis of the shoulder with or without a torn glenoid labrum.

Successful results can be achieved in approximately half the patients with partial tears of the rotator cuff, re-tears of the cuff after a previous repair, and adhesions of the biceps tendon to the undersurface of the rotator cuff.

Of patients with severe osteoarthritis of the shoulder, complete tears of the rotator cuff, or in whom previous operations on the rotator cuff have failed, only a few can be expected to benefit.
Arthroscopic synovectomy for rheumatoid arthritis and arthroscopically controlled fixation for instability may prove to be of value but the follow-up is not long enough.

Resection of the glenoid labrum in the presence of instability of the shoulder is contra-indicated; stabilisation is needed. Further research needs to be directed into arthroscopic release of rotator cuff impingement and the role of arthroscopically controlled stabilisation.

\section{REFERENCES}

Andrews JR, Carson WG. Shoulder joint arthroscopy. Orthopedics 1983:6:1157 62.

Cofield RH. Arthroscopy of the shoulder. Mayo Clinic Proc 1983:58: 5018.

Conti V. Arthroscopy in rehabilitation. Orthop (lin North Am 1979: 10(3): 70911 .

DePalma AF. Surgery of the shoulder. 3rd ed. Philadelphia: JB Lippincott. 1983.

Earnshaw P, Desjardins D, Sarkar K, Uhthoff HK. Rotator cuff tears: the role of surgery. Can J Surg 1982:25:60 3 .

Ha'eri GB, Maitland A. Arthroscopic findings in the frozen shoulder. $J$ Rheumatol 1981:8:149 52.

Ha'eri GB, Wiley AM. Advancement of the supraspinatus muscle in the repair of ruptures of the rotator cuff. J Bome' Joint Surg $[.4 \mathrm{~m}]$ 1981: $63 \mathrm{~A}: 2328$.

Hawkins RJ, Kennedy JC. Impingement syndrome in athletes. Am J Sports Med 1980;8(3): 151-8.

Lundberg BJ. The frozen shoulder. Acta Orthop Scand 1969: Suppl 119.

Loyd JA, Loyd HA. Adhesive capsulitis of the shoulder: arthrographic diagnosis and treatment. South Med J 1983:76:879 83.

Matthews LS, Vetter WL, Helfet DL. Arthroscopic surgery of the shoulder. Adr Orthop Surg 1984:7:203 10.

Moseley HF, Óvergaard B. The anterior capsular mechanism in recurrent anterior dislocation of the shoulder: morphological and clinical studies with special reference to the glenoid labrum and the gleno-humeral ligaments. J Bone Joint Surg $[\mathrm{Br}]$ 1962:44 B: 91327.

Neviaser JS. Adhesive capsulitis of the shoulder. Am Acad Orthop Surg Instr Course' Le't 1949:6:281 91.

Packer NP, Calvert PT, Bayley JIL, Kessel L. Operative treatment of chronic ruptures of the rotator cuff of the shoulder. J Bone Joint Surg $[B r] 1983: 65-B: 1715$.

Pappas AM, Goss TP, Kleinman PK. Symptomatic shoulder instability due to lesions of the glenoid labrum. Am J Sports Med 1983:11: 27988.

Petersson CJ. Degeneration of the gleno-humeral joint: an anatomical study. Acta Orthop Scand 1983:54:277 83.

Simmonds FA. Shoulder pain: with particular reference to the "frozen" shoulder. J Bone Joint Surg $[B r]$ 1949:31 B:426 32.

Wiley AM, Older MW. Shoulder arthroscopy: investigations with a fibreoptic instrument. Am J Sports Med 1980:8:31 8 . 